\title{
Treatment of temporomandibular disorders - knowledge, attitudes and clinical experience among general practising dentists in Sweden
}

\section{Erik Lindfors, Åke Tegelberg, Tomas Magnusson \& Malin Ernberg}

To cite this article: Erik Lindfors, Åke Tegelberg, Tomas Magnusson \& Malin Ernberg (2016) Treatment of temporomandibular disorders - knowledge, attitudes and clinical experience among general practising dentists in Sweden, Acta Odontologica Scandinavica, 74:6, 460-465, DOI: 10.1080/00016357.2016.1196295

To link to this article: http://dx.doi.org/10.1080/00016357.2016.1196295

Published online: 21 Jun 2016.

Submit your article to this journal $\llbracket$

山ll Article views: 154

View related articles ־

View Crossmark data $\asymp$ 


\title{
Treatment of temporomandibular disorders - knowledge, attitudes and clinical experience among general practising dentists in Sweden
}

\author{
Erik Lindfors ${ }^{\mathrm{a}, \mathrm{b}}$, Åke Tegelberg ${ }^{\mathrm{c}, \mathrm{d}, \mathrm{e}}$, Tomas Magnusson ${ }^{\mathrm{f}}$ and Malin Ernberg ${ }^{\mathrm{b}}$

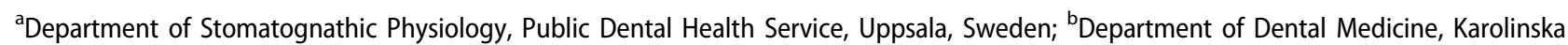 \\ Institutet, Scandinavian Center for Orofacial Neuroscience (SCON), Huddinge, Sweden; 'Department of Orofacial Pain and Jaw Function, \\ Faculty of Odontology, Malmö University, Malmö, Sweden; ${ }^{d}$ Clinical Centre of Research, Uppsala University, Västerås, Sweden; ${ }^{e}$ Postgraduate \\ Education Dental Center, Örebro, Sweden; 'School of Health and Welfare, Jönköping University, Jönköping, Sweden
}

\begin{abstract}
Objective: The aim of the study was to investigate the self-perceived level of knowledge, attitudes and clinical experience in treatment of temporomandibular disorders (TMD) among general practising dentists (GPDs).

Material and methods: A web-based questionnaire was sent to all GPDs in the public dental health service in the County of Uppsala in $2010(n=128)$ and $2014(n=113)$. The GPDs were asked to answer questions in the following categories: Demographic information, Quality assurance, Clinical experience and treatment, Need for specialist resources in the field of TMD and Attitudes. Between the two questionnaires, the GPDs were offered TMD education and an examination template including three TMD questions was introduced in the computer case files. The results were also compared with a previous questionnaire from 2001.

Results: The response rate was $71 \%$ (2010) and $73 \%$ (2014). The majority of the GPDs were women (70\% in 2010 and $72 \%$ in 2014). The reported frequency of taking a case history of facial pain and headache increased between 2010 and 2014. In 2014, the GPDs were more secure and reported higher frequency of good clinical routines in treatment with jaw exercises and pharmacological intervention compared to 2001. Interocclusal appliance was the treatment with which most dentists felt confident and reported good clinical routines.

Conclusions: The GPDs felt more insecure concerning TMD diagnostics, therapy decisions and treatment in children/adolescents compared to adults. There is a high need for orofacial pain/TMD specialists and a majority of the GPDs wants the specialists to offer continuing education in TMD.
\end{abstract}

\section{ARTICLE HISTORY}

Received 21 February 2016 Revised 16 May 2016

Accepted 27 May 2016 Published online 20 June 2016

\section{KEYWORDS}

Dentistry; jaw exercises; occlusal splint; orofacial pain; postgraduate education

\section{Introduction}

Temporomandibular disorders (TMD) are a group of conditions affecting the temporomandibular joints (TMJs) and masticatory muscles. Pain is one of the most common symptoms of TMD.[1] Over the years, different treatment modalities have been suggested in the management of TMD. These recommended therapies include different kinds of interocclusal appliances, occlusal adjustment, physiotherapy, jaw exercises, acupuncture, transcutaneus electric nerve stimulation, cognitive behavioural therapies and pharmacological interventions.[1-3] Earlier studies have shown that two of the most commonly used TMD treatments in general practice are interocclusal appliances and occlusal adjustment.[4-6] The treatment need of TMD in the adult population has been estimated to be between $5 \%$ and 15\%.[7] Accessible statistics form the Swedish health insurance show that only $0.5-1.5 \%$ of the adult population in Sweden receive TMD treatment.[7] Nilsson et al. [8] reported that $4.2 \%$ of children and adolescents experienced TMD pain, but only one-third of these had received treatment. These figures indicate an under-treatment of TMD both in adults and children/adolescents.

In an earlier study, Tegelberg et al. [9] concluded that a majority of general practising dentists (GPDs) in Sweden lacked routines in diagnostics, choosing therapy and evaluating treatment results in children and adolescents with TMD. In that study, good clinical routines were reported only for interocclusal appliance treatment. Dentists' knowledge and attitudes concerning TMD in adults have been investigated in previous studies [10-12] using the same postal questionnaire but in different geographical areas. These studies concluded that there is a large discrepancy between general dentists' and orofacial pain/TMD experts' opinions on the aetiology of $T M D$, diagnostics and choice of therapy.

The aims of the present study were to investigate if the self-perceived level of knowledge, attitudes and clinical experience in the treatment of children, adolescents and adults with TMD among GPDs, changed over time. It was also intended to document the need for specialist resources in the field of TMD.

\section{Materials and methods}

A web-based questionnaire was sent to all GPDs in the public dental health service in the County of Uppsala, Sweden $(n=128)$ in September 2010. GPDs who did not answer the questionnaire received an e-mail reminder after two weeks. 
A maximum of three reminders were sent. The questionnaire comprised 20 multiple-choice questions which were based on an earlier postal questionnaire by Tegelberg et al. in 2001.[9] The dentists were asked to answer questions in the following categories: Demographic information - gender; number of years in profession. Quality assurance - presence of health declaration containing questions on the topic of orofacial pain and headache; regular case history of orofacial pain and headache; participation in post-graduate TMD education. Clinical experience and treatment - self assessment of the GPDs own skills in diagnostics, therapy decision, various treatments and evaluation of treatment. The questions were answered using the following scale: 1 = lack of routine/unable, 2 = limited routine/unsure and 3 =good routine/confident. The GPDs were also asked to answer what kind of interocclusal appliances the GPDs commonly made; the three most common indications for interocclusal appliance treatment; clinical follow-up routines concerning interocclusal appliance treatment. Need for specialist resources in the field of TMD - need for consultation visits in their own clinic; need for telephone consultations; need for the possibility to refer patients to an orofacial pain/TMD specialist; need for the possibility to auscultate at a specialist clinic; need for post graduate education. Attitude - The dentists were asked to finish each of the two sentences 'To treat adults with TMD pain is...' and 'To treat children/adolescents with TMD pain is. .' with 2 of the 10 following adjectives: interesting, educational, rewarding, worthwhile, challenging, stressful, difficult, frustrating, unpleasant and demanding. The first 5 adjectives were judged to be positive and the last 5 to be negative. If both selected adjectives were positive, the attitude was judged to be positive. If one adjective was positive and the other one was negative, the attitude was judged to be neutral and, consequently, two negative adjectives were judged as a negative attitude.

During the time period January 2011 to February 2014, the specialist unit for orofacial pain/TMD offered the GPDs, in the public dental health service in Uppsala, 5 seminars, 7 short courses and 2 major courses in TMD. These educations were part of the strategic educational program at the public dental health service in the County of Uppsala.

In January 2011, an optional examination template was introduced in the computer case files containing the following three questions; 'Do you have pain in your temples, face, TMJs, or jaws once per week or more often?', 'Do you have pain when you open your mouth wide or when you chew once per week or more often?' and 'Do you experience locking or other functional problems when you chew or open your mouth once per week or more often?'

A second web-based follow-up questionnaire was sent to all GPDs in the public dental health service in the County of Uppsala $(n=113)$ in February 2014. Dentists who did not answer received an e-mail reminder according to the routines previously described. The follow-up questionnaire comprised 8 of the original 20 multiple-choice questions in these categories: Demographic information - gender. Quality assurance regular case history of orofacial pain and headache; participation in post-graduate TMD education. Clinical experience and treatment - self-assessment of the dentist's own ability in diagnostics, therapy decision, various treatments and evaluation of treatment. Attitude - the dentists were asked to finish each of these two sentences: 'To treat adults with TMD pain is...' and 'To treat children/adolescents with TMD pain is...' with 2 out of 10 adjectives (see above).

The results from the present study (2010 and 2014) were also compared with the results from a previous study by Tegelberg et al. [9] in order to analyse if there were any major changes over a longer period of time.

\section{Statistics}

The results are presented as frequencies and mean values. For the statistical analyses of differences between variables and groups, chi-square test was used (SigmaPlot). A $p$ value $<0.05$ was considered as a statistically significant difference.

\section{Ethical approval}

After correspondence with the regional ethical review board at Uppsala University, it was concluded that this study did not need an ethical vetting.

\section{Results}

\section{Demographics and response rate}

The response rate in the 2010 questionnaire was $71 \%$ $(n=91)$. In the follow-up questionnaire in 2014, the response rate was $73 \%(n=82)$. The majority of the dentists were women, 70\% $(n=64)$ in 2010, 72\% $(n=59)$ in 2014, as compared to $71 \%(n=177)$ in 2001 . The mean number of years in profession was 17.4 years both in 2010 (range: 1-39 years) and 2001. There was no statistical significant difference between the responders in respect of gender and working experience.

\section{Quality assurance}

In $2010,13 \%$ of the GPDs stated that they used a health declaration containing questions on facial pain and headache.

In 2010, 51\% of the GPDs stated that they had received continuing postgraduate education about TMD. At the followup in 2014, 35\% of the GPDs stated that they had received education about TMD during the time-period 2011-2014. A majority of these dentists (83\%) had attended the TMD education program offered by the public dental health service in the County of Uppsala.

An increase in the frequency of 'regular case history of facial pain and headache' was seen between 2010 and 2014 both in children/adolescents $(28 \%$ and $45 \%$, respectively, $p=0.027$ ) and in adults (70\% and $89 \%$, respectively, $p=0.004$ ). Both in 2010 and 2014 significantly fewer GPDs reported taking 'regular case history of facial pain and headache' in children/ adolescents compared to in adults $(p<0.001)$.

\section{Clinical experience and treatment}

Compared to 2001 fewer dentists reported in 2010 and 2014, respectively, that they had good routines and confidence in 
treating children/adolescents with interocclusal appliances and occlusal adjustment (Table 1). Still, interocclusal appliance treatment was the treatment alternative in which most of the GPDs felt that they had good clinical routines and confidence both in 2010 and 2014.

More GPDs felt that they had good clinical routine and confidence when treating children/adolescents with therapeutic jaw exercises in 2010/2014 compared to 2001. The dentists also felt more confident in pharmacological intervention in children/adolescents in 2014 compared to 2001 and 2010 (Table 1).

There were no statistically significant changes concerning diagnostics, therapy decision and evaluation of treatment results in children/adolescents over time (2001-2010-2014). In general, few GPDs reported good clinical routines and confidence concerning these variables (Table 1).

The GPDs felt more insecure concerning TMD diagnostics, therapy decisions and treatment with interocclusal appliance, jaw exercises and pharmacological intervention in children/ adolescents than in adults (Table 1). The GPDs felt more confident regarding pharmacological intervention in adults in 2014 compared to 2010 (Table 1).

The dentists reported that they choose stabilization appliances in $24 \%$ of the cases in children/adolescents and in $97 \%$ of the adult cases. The corresponding figures for soft appliances were $74 \%$ and $2 \%$, respectively. In 2010, $91 \%$ of the GPDs reported that they used to follow-up interocclusal appliance treatment with clinical controls.

In 2010, the three most common indications for treatment with interocclusal appliances reported by the GPDs were (in the following order): Pain from the masticatory system, Tension-type headache and Tooth wear due to bruxism.

\section{Discussion}

\section{Demographics and response rate}

In a large comprehensive review, concerning the response rate of general practicing physicians to postal questionnaires, it was concluded that the overall response rate was 61\%.[13] The response rates in the present study, 71\% in 2010 and $73 \%$ in 2014, must therefore be considered good for a questionnaire study. The advantage of the present questionnaire study is that it is based on an earlier postal questionnaire from 2001 [9] and that it has a cross sectional follow-up design. In this way, comparisons could be made over a long period of time (2001-2014). The agreement concerning the demographic factors gender and number of years in profession in the present questionnaire study and the earlier study [9] was very good. The fact that the earlier study [9] was conducted in other counties, with other possible treatment traditions, should be taken under consideration when the results are interpreted. A disadvantage of the study, as already mentioned by Tegelberg et al.,[9] is concerning the questions' validity and reliability which has not been investigated.

Figures from the Swedish Association of Local Authorities and Regions,[14] the Swedish Association of Public Dental Officers [15] and the National Board of Health and Welfare [16] concerning gender (67.8\% women), working experience (mean 17.1 years) and age distribution of Swedish dentists in the public dental health care on a national level correspond well with the responders in the present study. Thus, the responders in the present study can be considered representative for dentists in the public dental health care on a national level.

A weakness of the follow-up part of the material is that it is not known if the responders in the two questionnaires (2010 and 2014) were the same. We can therefore not treat the results as longitudinal prospective data. Still, a relative low turnover on GPDs in the public dental health service in the County of Uppsala and the high response rate to both questionnaires lend strength to the results and allow for comparison between the questionnaires.

\section{Quality assurance}

In 2010 , only $13 \%$ of the dentists reported that they used a health declaration containing questions on facial pain and

Table 1. Self-evaluation of clinical experience and skill concerning good routines and confidence in TMD diagnostics, therapy decision, performance of different treatments and assessment of treatment results in children/adolescents and adults with TMD.

\begin{tabular}{|c|c|c|c|c|c|c|}
\hline & \multicolumn{2}{|c|}{2014 (County I) $n=82$} & \multicolumn{2}{|c|}{2010 (County I) $n=91$} & \multirow{2}{*}{$\frac{2001\left(\text { County II-IV) }{ }^{\mathrm{a}} n=250\right.}{\text { Children/adolescents }}$} & \multirow[b]{2}{*}{$p$ Value } \\
\hline & Children/adolescents & Adults & Children/adolescents & Adults & & \\
\hline Diagnostics & $27(32)$ & $44(54)$ & $25(27)$ & $43(47)$ & $89(36)$ & $\begin{array}{l}0.002^{\mathrm{A}} \\
0.0012^{\mathrm{B}}\end{array}$ \\
\hline Interocclusal appliance & $49(60)$ & $68(83)$ & $64(70)$ & $75(82)$ & $195(78)$ & $\begin{array}{l}0.002^{B} \\
0.005^{C}\end{array}$ \\
\hline Occlusal adjustment & $15(18)$ & $23(28)$ & $17(19)$ & $16(18)$ & $105(42)$ & $<0.001^{\mathrm{C}}$ \\
\hline Pharmacological intervention & $11(13)$ & $25(30)$ & $3(3)$ & $12(13)$ & $16(6)$ & $\begin{array}{l}0.031^{\mathrm{A}} \\
0.014^{\mathrm{B}} \\
0.029^{\mathrm{D}} \\
0.033^{\mathrm{E}} \\
0.011^{\mathrm{F}}\end{array}$ \\
\hline Evaluation of treatment results & $28(34)$ & $38(46)$ & $20(22)$ & $31(34)$ & $75(30)$ & NS \\
\hline
\end{tabular}

Comparison between different years and groups. Figures express number of responders (percentage distribution within brackets). A = between children/adolescents and adults in 2010, B = between children/adolescents and adults in 2014, C= between children/adolescents in 2001 and 2010/2014, D= between children/adolescents in 2001 and 2014, $\mathrm{E}=$ between children/adolescents in 2010 and 2014, F= between adults in 2010 and 2014

County I= County of Uppsala.

County II-IV = Counties of Östergötland, Västmanland and Göteborg

Data from Tegelberg et al. [9] 
headache. Due to a probable under-treatment in both adults and children/adolescents, $[7,8]$ it is important to enhance the identification of these patients. It has been suggested that standardized questions in a health declaration could improve the detection of patients with TMD pain.[9] Even though a majority of dentists did not have specific questions about TMD pain in a health declaration in $2010,70 \%$ reported that they took regular case histories of facial pain and headache in their adult patients. The corresponding figure in children/ adolescents was much lower; $28 \%$. One explanation for this might be that, in Sweden, children and adolescents are commonly examined by dental hygienists or dental assistants, and that the GPDs therefore do not ask the patients about this anamnestic information. In 2014, the proportion of dentists who reported that they took regular case histories of facial pain and headache had increased both in adults and children/adolescents. This increase might be the result of the introduction of questions about TMD pain in the optional examination template in the computer case files. Nilsson et al. [17] have shown that these questions have a good reliability and validity in adolescents. Another factor that might have influenced the increased frequency is the strategic educational TMD program in the public dental health service in Uppsala County. Still, definite conclusions concerning such a connection is not possible to make due to study design and the lack of true longitudinal prospective data. In 2010, half of the GPDs reported that they had attended postgraduate education in TMD and in 2014, one-third reported further continuing education in TMD (mainly by attending the internal educational program in TMD). Education and training have been shown to increase the adoption rate of new treatment technique and the frequency of good-quality care in dentistry.[18] Continuing postgraduate TMD education is of probable importance to increase the identification of TMD patients and to improve patients' care.

\section{Clinical experience and treatment}

A majority of the GPDs reported that they had good clinical routines and confidence in interocclusal appliance treatment both in adults and in children/adolescents. This finding is not surprising since earlier studies [4-6] have shown that this treatment is one of the most commonly used TMD therapies. Still, fewer GPDs reported that they had good clinical routines and confidence in interocclusal appliance treatment in children/adolescents in 2010 and 2014 compared to 2001.

In 2010 and 2014, fewer GPDs also reported that they had a good clinical routine and confidence in occlusal adjustments in children/adolescents compared to 2001.[9] Occlusal adjustment has been questioned as a TMD therapy for many years. $[19,20]$ To perform reversible TMD treatments is the predominant treatment concept in Scandinavia.[2] These two facts might have influenced and reduced the frequency of occlusal adjustment performed and thereby also the selfreported frequency of good clinical routine and confidence for this treatment. Also in adults, a majority of dentists reported that they felt insecure and did not have good clinical routines in occlusal adjustment. According to the National
Guidelines for Adult Dental Care,[7] there are still indications for occlusal adjustment in the treatment of some types of TMD patients. It is therefore a problem that a majority of GPDs reported that they lack good clinical routines and confidence in this treatment modality.

Concerning jaw exercises and pharmacological intervention (i.e., mostly analgesics and NSAIDs), the opposite trend was found. The proportion of GPDs that reported good clinical routines and confidence in jaw exercises when treating children/adolescents increased over time. This corroborates well with the already mentioned Scandinavian concept of reversible TMD treatments.[2] A small, but statistically significant, increase in the frequency of good clinical routines and confidence in pharmacological intervention in children/adolescents was also seen over time. Still, the great majority of GPDs reported that they lacked good clinical routines and confidence in this treatment. Again, postgraduate continuing education in different kinds of TMD treatments is probably important.

The GPDs felt more insecure concerning TMD diagnostics, therapy decisions and treatment with interocclusal appliance, jaw exercises and pharmacological intervention in children/ adolescents than in adults. During the years 2009 to 2014, the incidence of interocclusal appliances in adults increased, whereas the incidence in children/adolescents did not change.[21] One can speculate that the more patients a dentist examines and treats, the more confident and skilled the dentist gets. This might in part explain that the GPDs felt more secure in the diagnostics and treatment of adult TMD patients. In 2010, $13 \%$ of the GPDs reported good clinical routine and confidence in pharmacological intervention of adults with TMD. The corresponding figure in 2014 was 30\%. The internal strategic educational program in TMD might partly explain this increase.

A majority of GPDs wanted to have the possibility to consult or refer TMD patients to an orofacial pain/TMD specialist (Table 2). The complexity of TMD, self-perceived lack of knowledge and the feeling that TMD treatment is non-profitable might be some factors that can explain this demand. A majority of the GPDs also wanted to use the specialist for continuing education and for acquiring new knowledge (Table 2). The figures from 2010 were almost identical to the figures reported in 2001.[9] This means that the high need

Table 2. How the general practising dentists wish to use specialist resources in orofacial pain/TMD.

\begin{tabular}{lcc}
\hline & $\begin{array}{c}2010 \text { (County I) } \\
n=91\end{array}$ & $\begin{array}{c}2001 \text { (County II-IV) }^{\text {a }} \\
n=250\end{array}$ \\
\hline $\begin{array}{l}\text { Consultation in the clinic of the general } \\
\text { practising dentist }\end{array}$ & $66(73)$ & $173(69)$ \\
$\begin{array}{l}\text { Consultation and referral of patients for } \\
\text { treatment }\end{array}$ & $87(96)$ & $248(99)$ \\
$\begin{array}{l}\text { Auscultation or clinical work with a oro- } \\
\text { facial pain/TMD specialist }\end{array}$ & $63(69)$ & $180(72)$ \\
$\begin{array}{l}\text { Continuing education and/or new } \\
\text { knowledge }\end{array}$ & $84(92)$ & $243(97)$ \\
\hline
\end{tabular}

Comparison between 2010 and 2001. There were no significant differences between the two years. Figures express number of responders (percentage distribution within brackets).

County I = County of Uppsala.

County II-IV = Counties of Östergötland, Västmanland and Göteborg.

${ }^{\mathrm{a}}$ Data from Tegelberg et al. [9] 
for orofacial pain/TMD specialists has been solid over a long period of time.

\section{Attitude}

A majority of the GPDs were positive to treat adults and about half of them were positive to treat children/adolescents. There were no statistically significant changes concerning attitudes over time (Table 3). Attitudes of the GPDs have been suggested to be the most important factor in the guidance of care.[9] Attitudes are probably better investigated with a qualitative research method, for example a focus group study, than by a questionnaire study. The validity of the questions used in this study can be questioned. Still, as mentioned earlier, the strength of this study lies in the crosssectional follow-up design as well as in the fact that the data can be compared with earlier studies.

The most common type of interocclusal appliance made for adults was the stabilization appliance and in children/adolescents, the soft appliance was as common as the stabilization appliance (Table 4).[21] The main reason for this difference is probably that children are still growing and that the dentists do not want to hamper the development of the jaws and teeth in younger children. One might also speculate that since a soft appliance is cheaper than a stabilization appliance, more soft appliances are made for children/adolescents, who, in Sweden, have free dental care.

The incidence of TMD interventions in adults, on a national level, was $0.69 \%$ in 2014 . This figure was stable during the years 2010-2014.[16,22] In 2014, the incidence of occlusal appliances in adults, in the public dental health service, Uppsala, was $0.90 \%$.[21] The corresponding figure during the time period 2000-2002 in Uppsala was 0.66\%.[6] This increase in incidence might be explained by several factors. First, the self-reported frequency of bruxism has been shown to increase over time.[23] Thus, the increased frequency of stabilization appliances made might in part be explained by a higher patient demand for this treatment. A second possible explanation is that more TMD patients are detected due to increased knowledge about TMD among the GPDs, and also the use of more structured anamnestic TMD pain questions. The strategic internal educational program in TMD and the incorporation of TMD questions in the case file template might be of some importance in this aspect. A third explanation is that the number of patients choosing regular dental care at a fixed annual fee, in which also interocclusal appliance treatment is included, has increased. In 2014, the incidence of interocclusal appliances in this group of patients was $50 \%$ higher than in regular paying adults.[21] The group with dental care at a fixed annual fee increased substantially in the County of Uppsala between 2009 and 2014 and therefore the number of occlusal appliances made also increased.

The three most common indications for interocclusal appliance treatment reported by the GPDs in the 2010 questionnaire were: Pain from the masticatory system, Tension-type headache and Tooth wear due to bruxism. Lindfors et al. have previously shown, both in a case file study [6] and a postal questionnaire study,[24] that these are the most common indications for interocclusal appliance treatment in Sweden.

It is concluded that the GPDs felt more insecure concerning TMD diagnostics, therapy decisions and treatment in children/adolescents compared to adults. Most GPDs reported that they felt confident and had good clinical routines in interocclusal appliance therapy. Finally, there is a high need for orofacial pain/TMD specialists and a majority of the GPDs wants the specialists to offer continuing education in TMD.

Table 3. General practising dentists' attitudes to the care of children/adolescents and adults with TMD.

\begin{tabular}{|c|c|c|c|c|c|c|}
\hline \multirow[b]{2}{*}{ Attitude } & \multicolumn{2}{|c|}{2014 (County I) $n=82$} & \multicolumn{2}{|c|}{2010 (County I) $n=91$} & \multirow{2}{*}{$\frac{2001 \text { (County II-IV) }{ }^{\mathrm{a}} n=250}{\text { Children/adolescents }}$} & \multirow[b]{2}{*}{$p$ Value } \\
\hline & Children/adolescents & Adults & Children/adolescents & Adults & & \\
\hline Positive & 45 & 59 & 46 & 59 & 55 & NS \\
\hline Neutral & 33 & 28 & 41 & 28 & 39 & NS \\
\hline No answer & 10 & 4 & 0 & 0 & 0 & - \\
\hline
\end{tabular}

An association test where 2 of 10 possible adjectives (5 positive and 5 negative) where chosen. Comparison between; (1) children/adolescents and adults in 2010 and 2014, and (2) children/adolescents in 2001, 2010 and 2014 are presented. Figures show distribution in percentage (\%).

Positive $=$ both adjectives chosen were positive.

Neutral $=$ of the adjectives chosen, one was positive and one was negative.

Negative $=$ both adjectives chosen were negative.

County I= County of Uppsala.

County II-IV = Counties of Östergötland, Västmanland and Göteborg

${ }^{\mathrm{a}}$ Data from Tegelberg et al. [9]

Table 4. The number of soft and stabilization appliances made in the public dental health service in the County of Uppsala, 2009-2014.[22]

\begin{tabular}{|c|c|c|c|c|c|c|}
\hline \multirow{2}{*}{ Year } & \multicolumn{3}{|c|}{ Children/Adolescents (I + II) } & \multicolumn{3}{|c|}{ Adults (III + IV) } \\
\hline & Soft appliance (I) & Stabilization appliances (II) & Total $(I+I I)$ & Soft appliance (III) & Stabilization appliances (IV) & Total (III + IV) \\
\hline 2009 & $173(53)$ & $153(47)$ & $326(100)$ & $156(22)$ & $563(78)$ & 719 (100) \\
\hline 2010 & $160(55)$ & $131(45)$ & $291(100)$ & $170(18)$ & 791 (82) & $961(100)$ \\
\hline 2012 & $121(43)$ & $161(57)$ & $282(100)$ & $215(17)$ & $1030(83)$ & $1245(100)$ \\
\hline 2013 & 119 (42) & $166(58)$ & $285(100)$ & 173 (14) & 1037 (86) & $1210(100)$ \\
\hline 2014 & $125(47)$ & $143(53)$ & $268(100)$ & $171(16)$ & 917 (84) & $1088(100)$ \\
\hline
\end{tabular}

Figures express number of interocclusal appliances (percentage distribution within brackets). 


\section{Acknowledgements}

We would like to thank all the dentists who, without any economical or other benefits, participated in this study.

\section{Disclosure statement}

The authors report no conflicts of interest. The authors alone are responsible for the content and writing of this article.

\section{Funding information}

The study has received financial support from the Public Dental Health Service, Uppsala, Sweden.

\section{Notes on contributors}

Erik Lindfors, DDS, Senior consultant, Specialist in Stomatognathic Physiology, Uppsala, Sweden.

Åke Tegelberg, DDS, PhD, Senior consultant in Orofacial pain and jaw function, professor of faculty of Odontology, Malmö university, Malmö, Sweden.

Tomas Magnusson, DDS, PhD, Specialist in Stomatognathic Physiology, Professor emeritus in Oral Health Sciences, School of Health and Welfare, Jönköping University, Sweden.

Malin Ernberg, DDS, PhD, Senior consultant, Specialist in Stomatognathic Physiology, Professor in Clinical Oral Physiology.

\section{References}

[1] Okeson JP, editor. Orofacial pain: guidelines for assessment, diagnosis \& management, American Academy of Orofacial Pain. 3rd ed. Chicago (IL): Quintessence Publishing; 1996.

[2] Carlsson GE, Magnusson T. Management of temporomandibular disorders in the general dental practice. Chicago (IL): Quintessence Publishing; 1999. p. 93-121.

[3] Shedden Mora MC, Weber D, Neff A, et al. Biofeedback-based cognitive-behavioral treatment compared with occlusal splint for temporomandibular disorder: a randomized controlled trial. Clin J Pain 2013;29:1057-1065.

[4] Glass EG, Glaros AG, McGlynn FD. Myofascial pain dysfunction: treatments used by ADA members. J Craniomandib Pract. 1993; 11:25-29.

[5] Glass EG, McGlynn FD, Glaros AG. A survey of treatments for myofascial pain dysfunction. J Craniomandib Pract. 1991; 9:165-168.
[6] Lindfors E, Magnusson T, Tegelberg A. Interocclusal appliances indications and clinical routines in general dental practice in Sweden. Swed Dent J. 2006;30:123-134.

[7] National Guidelines for Adult Dental Care. The National Board of Health and Welfare; 2011, p. 12, 185, 190, 193, 195, 205.

[8] Nilsson IM, List T, Drangsholt M. Prevalence of temporomandibular pain and subsequent dental treatment in Swedish adolescents. J Orofac Pain 2005;19:144-150.

[9] Tegelberg A, List T, Wahlund K, et al. Temporomandibular disorders in children and adolescents: a survey of dentists' attitudes, routine and experience. Swed Dent J. 2001;25:119-127.

[10] Glaros AG, Glass EG, McLaughlin L. Knowledge and beliefs of dentists regarding temporomandibular disorders and chronic pain. Orofac Pain 1994;8:216-222.

[11] Le Resche L, Truelove EL, Dworkin SF. Temporomandibular disorders: a survey of dentists' knowledge and beliefs. J Am Dent Assoc. 1993;124:90-94, 97-106.

[12] Lee WY, Choi JW, Lee JW. A study of dentists' knowledge and beliefs regarding temporomandibular disorders in Korea. J Craniomandib Pract. 2000;18:142-146.

[13] Creavin ST, Creavin AL, Mallen CD. Do GPs respond to postal questionnaire surveys? A comprehensive review of primary care literature. Fam Pract. 2011;28:461-467.

[14] Swedish Association of Local Authorities and Regions. Table 6 LP 2014.

[15] The Swedish Association of Public Dental Officers. Member register.

[16] The National Board of Health and Welfare. Statistical database.

[17] Nilsson IM, List T, Drangsholt M. The reliability and validity of selfreported temporomandibular disorder pain in adolescents. J Orofac Pain 2006;20:138-144.

[18] Dahlström L, Molander A, Reit C. The impact of a continuing education programme on the adoption of nickel-titanium rotary instrumentation and root-filling quality amongst a group of Swedish general dental practitioners. Eur J Dent Educ. 2015;19:23-30.

[19] Koh H, Robinson PG. Occlusal adjustment for treating and preventing temporomandibular joint disorders. J Oral Rehabil. 2004;31:287-292.

[20] List T, Axelsson S. Management of TMD: evidence from systematic reviews and meta-analyses. J Oral Rehabil. 2010;37:430-451.

[21] Public Dental Health Service, Uppsala, Sweden. Database register.

[22] Statistics Sweden. Statistical database - Population - Population statistics - Number of inhabitants.

[23] Anastassaki Köhler A, Hugoson A, Magnusson T. Prevalence of symptoms indicative of temporomandibular disorders in adults: cross-sectional epidemiological investigations covering two decades. Acta Odontol Scand. 2012;70:213-223.

[24] Lindfors E, Helkimo M, Magnusson T. Patients' adherence to hard acrylic interocclusal appliance treatment in general dental practice in Sweden. Swed Dent J. 2011;35:133-142. 